\title{
Developing Advanced Human Support Technologies for Planetary Exploration Missions
}

\author{
Debra P. Berdich* and Paul D. Campbell ${ }^{\dagger}$ \\ Lockheed Martin Space Operations, Houston, Texas, 77058 \\ J. Mark Jernigan ${ }^{\ddagger}$ \\ National Aeronautics and Space Administration Lyndon B. Johnson Space Center, Houston, Texas, 77058
}

\begin{abstract}
The United States Vision for Space Exploration calls for sending robots and humans to explore the Earth's moon, the planet Mars, and beyond. The National Aeronautics and Space Administration (NASA) is developing a set of design reference missions that will provide further detail to these plans. Lunar missions are expected to provide a stepping stone, through operational research and evaluation, in developing the knowledge base necessary to send crews on long duration missions to Mars and other distant destinations. The NASA Exploration Systems Directorate (ExSD), in its program of bioastronautics research, manages the development of technologies that maintain human life, health, and performance in space. Using a systems engineering process and risk management methods, ExSD's Human Support Systems (HSS) Program selects and performs research and technology development in several critical areas and transfers the results of its efforts to NASA exploration mission/systems development programs in the form of developed technologies and new knowledge about the capabilities and constraints of systems required to support human existence beyond Low Earth Orbit. HSS efforts include the areas of advanced environmental monitoring and control, extravehicular activity, food technologies, life support systems, space human factors engineering, and systems integration of all these elements. The HSS Program provides a structured set of deliverable products to meet the needs of exploration programs. These products reduce the gaps that exist in our knowledge of and capabilities for human support for long duration, remote space missions. They also reduce the performance gap between the efficiency of current space systems and the greater efficiency that must be achieved to make human planetary exploration missions economically and logistically feasible. In conducting this research and technology development program, it is necessary for HSS technologists and program managers to develop a common currency for decision making and the allocation of funding. A high level assessment is made of both the knowledge gaps and the system performance gaps across the program's technical project portfolio. This allows decision making that assures proper emphasis areas and provides a key measure of annual technological progress, as exploration mission plans continue to mature.
\end{abstract}

\section{Introduction}

$\mathrm{T}$ HE NASA is focused on the Vision for Space Exploration, a long-term effort to extend robotic and human missions across the Solar system. The HSS Program is a vital part of achieving this vision.

\section{A. Program Background and History}

The HSS Program was created by the NASA Office of Biological and Physical Research (OBPR) in the 1990's. Originally, the program grew by consolidation of a set of heritage projects, including the Closed Ecological Life Support System (CELSS) Program. In 1997, the National Research Council's Committee on Advanced Technology for Human Support in Space issued a report to NASA with recommendations on strengthening the HSS Program and

\footnotetext{
${ }^{*}$ Project Engineer, Mission Management Department, 2400 NASA Parkway, Mail Stop C46

${ }^{\dagger}$ Project Manager, Mission Management Department, 2400 NASA Parkway, Mail Stop C46

¥ Manager, Human Support Technology, Office of Bioastronautics, 2101 NASA Parkway, Mail Stop SA12
} 
improving the linkage between the human support and human health aspects of long duration space flight. ${ }^{1}$ HSS Program management was assigned to the Johnson Space Center (JSC), and it has been managed to date within the JSC Space and Life Sciences Directorate, by the Office of Bioastronautics.

\section{B. Program Context and Content}

The Bioastronautics Program encompasses human health research and countermeasure development, advanced space medicine technologies, radiation research, and human support systems technology development. This program conducts research in both Earth-based settings and in space flight onboard the International Space Station (ISS). ISS crewmembers serve as both researchers and as human subjects, to generate new knowledge about human adaptation to space and human performance capabilities in space environments. Knowledge and technology developed under this program is targeted to serve the needs of NASA's human space exploration programs.

HSS is a major part of the Bioastronautics Program. The HSS Program performs research and technology development to provide the basis for next-generation systems that enable humans to live and work safely and effectively in space. Special emphasis is placed on technologies that have a dramatic impact in the reduction of human logistical requirements, spacecraft mass, power, thermal, volume, and crew workload, and technologies that provide significantly increased mission safety and/or reliability.

The Program includes a set of related technology areas: environmental monitoring and control; extravehicular activity systems; food systems; life support systems; fire prevention, detection, and suppression; in-situ fabrication and repair; in-situ resource utilization; low-gravity and exploration research; and space human factors engineering. Each of these elements manages peer-reviewed research and technology development projects that are conducted by both external investigators and within NASA. Table 1 lists the HSS Program elements.

Table 1. HSS Program Elements

\begin{tabular}{|l|}
\hline Advanced Environmental Monitoring and Control (AEMC) \\
\hline Advanced Extravehicular Activity (AEVA) \\
\hline Advanced Food Technology (AFT) \\
\hline Space Human Factors Engineering (SHFE) \\
\hline Advanced Life Support (ALS) \\
\hline Fire Prevention, Detection, and Suppression (FPDS) \\
\hline In-Situ Fabrication and Repair (ISFAR) \\
\hline In-Situ Resource Utilization (ISRU) \\
\hline Low-Gravity and Exploration Research \\
\hline Systems Engineering and Integration (SE\&I) \\
\hline
\end{tabular}

Ground investigations are conducted in university and industry laboratories and at multiple NASA centers. HSS is also responsible for systems engineering and integration to assure technologies developed meet exploration vehicle and habitat needs and constraints. Currently, the Bioastronautics Laboratory at JSC is used as a site for integration of HSS technologies, but it is expected that higher fidelity ground analogs as well as space flight experiments will be required for the program to meet its commitment to deliver mature technologies to the exploration vehicle development programs. The program identifies and integrates externally developed technologies, where advantageous, via its Technology Integration Agent function.

Flight experiments are conducted onboard the existing programs, predominantly ISS and Shuttle. Flight systems integration and flight experiment operations are conducted via a Flight Integration function.

The HSS Program also collaborates with related research programs in areas such as life sciences, physical sciences, and advanced technologies, to maximize the value of the collective efforts and take advantage of known expertise. HSS results are provided to NASA human space flight programs to meet their technology needs, for application to the operational human programs and future human spacecraft. The main purpose of the program is to significantly reduce the programmatic technological and logistical risks to the missions defined by the NASA Exploration Systems Directorate. In addition, since its mission is human support, it is expected that HSS will develop technologies of direct benefit to the public and earth ecology as well as conducting deliberate education and outreach efforts that contribute to NASA's mission to inform and benefit the U.S. public and inspire the next generations.

\section{Program and Project Management}

The HSS Program uses an integrated set of management practices to provide program direction and control. The management of specific technical tasks is delegated to the program elements. These elements follow common NASA developed processes, ensuring that programmatic decisions are well-formulated and rational and that ultimate outcomes are traceable. 


\section{A. Strategic Alignment}

The NASA Strategic Plan ${ }^{2}$ includes high-level goals that guide ExSD and HSS. Goal 7, to "Engage the public in shaping and sharing the experience of exploration and discovery" is carried out through HSS education and outreach activities. Goal 9, to "Extend the duration and boundaries of human space flight to create new opportunities for exploration and discovery" provides the central focus for HSS technology development efforts. In response to this goal, ExSD is "...developing advanced human support technology that will enable dramatic reductions in mass, power, and volume, while increasing safety, reliability, and the availability of the crew to conduct scientific experiments." HSS is the primary means to achieve the NASA Strategic Plan's objective to "Develop knowledge and technologies to make life support systems self-sufficient and improve human performance in space."

The Biological \& Physical Research Enterprise Strategy ${ }^{3}$ frames the scope of bioastronautics programs by articulating a structured set of organizing questions. Question 4, "What technology must we create to enable the next explorers to go beyond where we have been?" provides the theme for HSS. Sub-question 4a, "How can we enable the next generation of autonomous, reliable spacecraft human support subsystems?" sets the stage for life support and food technology development. Sub-question 4c, "How can we enable optimum human performance and productivity during extended isolation from Earth?" motivates space human factors engineering research, and subquestion $4 \mathrm{~d}$, "What automated sensing and control systems must we create to ensure that the crew is living in a safe and healthy environment?" leads to the environmental monitoring and control work within HSS.

The Bioastronautics Strategy ${ }^{4}$ provides more specific programmatic goals that link directly to the HSS Program. Goal 1, "Managing Risk" directs HSS to identify critical risks, assess their impacts, establish acceptable levels of risk, and apply benefit/cost analysis in its technology development efforts. In meeting this goal, HSS reduces the programmatic risks associated with development of vehicle/habitat systems for expanding human presence in the solar system by increasing knowledge and maturing technologies. This reduction of risk entails the development of capabilities for in-situ detection, analysis, and control of contaminants, life support performance and safety investigations, and system design and robustness research. HSS searches for emergent complex system behavior and determines the reliability and robustness of designs, and identifies maintainability issues.

Goal 2, "Increasing Efficiency" specifies that HSS will develop technologies with the potential to reduce life support system mass by a factor of three by 2010 , decrease crew time requirements, and develop advanced technologies for environmental monitoring and control. HSS develops methods to reduce key constrained mission resources needed for human life sustenance. This is accomplished by development of technologies that close resource loops, such as air, water, and food; reduce the size of material buffers and the reliance on consumables, result in miniaturization of sensing and analytical capabilities onboard the spacecraft, and create improvements in crew productivity for mission operations. Integration of multi-component loops with sensors and controls increases autonomy and efficacy. HSS enables systems for sustaining the crew and assuring that the vehicle design is compatible with human physical and cognitive capabilities and limitations.

In early 2004, based on a White House initiative, the Vision for Space Exploration (VSE) was released by NASA $^{5}$. This vision laid out a broad plan for an extended program of robotic and human exploration of the Earth's moon, the planet Mars, and beyond. A Presidential commission was chartered to make recommendations on the implementation of the VSE. The commission report issued in June 2004 provided a description of enabling technologies to accomplish the VSE, including "Closed-loop life support and habitability - Recycling of oxygen, carbon dioxide, and water for long-duration human presence in space", "Extravehicular activity systems - the spacesuit of the future, specifically for productive work on planetary surfaces", and "Autonomous systems and robotics - to monitor, maintain, and where possible, repair complex space systems." ${ }^{6}$

HSS then participated in exploration alignment activities, to ensure that the program fully supports this new vision. HSS made initial contacts with NASA's exploration program to evaluate the priorities of specific HSS technical areas. While the NASA agency-level focus has crystallized, the HSS Program has aligned its content to develop human support technologies that enable exploration beyond low Earth orbit. 


\section{B. Risk Management}

HSS's purpose

is to reduce the

human support

systems

development risks

Table 2. Programmatic Risks Mitigated by the HSS Program

borne by the

exploration

programs to an acceptable level. The risks HSS addresses fall into three categories: (1) Risks to the safety and health of the crew and mission success due to the hazardous environment, autonomy, and isolation; (2) Risks to affordability of the missions by requiring too much logistical support Human Health and Safety

Robust provision of air, water, and food

In-situ detection and control of contaminants to human consumables

Ability to operate inside and out of the habitats to repair or mitigate system failures

Prevention or control of contingency situations (fire, toxic spill, etc)

Ensure mission systems and operations are designed within capabilities and constraints of crew and support team.

Reduce the human ecosystem footprint

Close loops to reduce buffers or re-supply needs for air, water, and food

Reduce or eliminate system consumables

Reduce mass, power, crew time, thermal load, and volume of in scope systems

Actively work with the ExSD to define capability needs and communicate HSS constraints

for the humans: (3)

\section{System Characteristics}

Investigate methods of in-situ maintenance

Simplify

Increase robustness

Develop diverse solutions to prevent monolithic failures

Discover and eliminate undesired complex system behavior of human support systems.

Risks to the human support systems in terms of system characteristics such as operability, reliability, and maintainability. Each risk is further characterized by research enabling questions. Acceptable mitigation through development of products that answer the enabling questions is required for all of the types of risks. Table 2 describes the three areas of programmatic risk.

The Bioastronautics Critical Path Roadmap (BCPR) is the principal risk management tool used by $\mathrm{HSS}^{7}$. A major revision of the BCPR was developed during 2003 and early 2004. This expanded the BCPR to cover all HSS program elements. Updated Design Reference Missions (DRM) were developed that are consistent with the VSE. The draft BCPR document was issued by NASA in April 2004 for review by the National Academies/Institute of Medicine (IOM), in collaboration with the National Academies' Division on Engineering and Physical Sciences (DEPS). This study

Table 3. BCPR Technical Risks Addressed by the HSS Program.

\begin{tabular}{|c|c|c|}
\hline Risk & Discipline & Title \\
\hline 36 & AEMC & Monitor Air Quality \\
\hline 37 & AEMC & Monitor External Environment \\
\hline 38 & AEMC & Monitor Water Quality \\
\hline 39 & AEMC & Monitor Surfaces Food and Soil \\
\hline 40 & AEMC & $\begin{array}{l}\text { Provide Integrated Autonomous Control of Life } \\
\text { Support Systems }\end{array}$ \\
\hline 41 & AEVA & $\begin{array}{l}\text { Provide Space Suits and Portable Life Support } \\
\text { Systems }\end{array}$ \\
\hline 42 & AFT & Maintain Food Quantity and Quality \\
\hline 43 & ALS & Maintain Acceptable Atmosphere \\
\hline 44 & ALS & Maintain Thermal Balance in Habitable Areas \\
\hline 45 & ALS & Manage Waste \\
\hline 46 & ALS & $\begin{array}{l}\text { Provide and Maintain Bioregenerative Life } \\
\text { Support Systems }\end{array}$ \\
\hline 47 & ALS & Provide and Recover Potable Water \\
\hline 48 & HST & $\begin{array}{l}\text { Inadequate Mission Resources for the Human } \\
\text { System }\end{array}$ \\
\hline 29 & SHFE & $\begin{array}{l}\text { Mismatch between Crew Cognitive Capabilities } \\
\text { and Task Demands }\end{array}$ \\
\hline 49 & SHFE & $\begin{array}{l}\text { Mismatch between Crew Physical Capabilities } \\
\text { and Task Demands }\end{array}$ \\
\hline 50 & SHFE & $\begin{array}{l}\text { Mis-assignment of Responsibilities within Multi- } \\
\text { agent Systems }\end{array}$ \\
\hline
\end{tabular}

4

American Institute of Aeronautics and Astronautics 
will focus on the content and processes currently used for communication, assessment, management, and implementation of the roadmap with respect to bioastronautics research for the missions contemplated in the VSE: extended human expeditions to the moon beginning between 2015-2020 and later human missions to Mars. Results of the study will include: (1) a comprehensive assessment and report of the strengths and weaknesses of the content and processes of the BCPR as applied to the missions laid out in the VSE; and (2) identification of unique challenges for accomplishing its goals and objectives. ${ }^{8}$ After this review, the BCPR will be baselined in 2005 .

The draft BCPR document contains the current applicable set of risks for the HSS Program that must be mitigated to enable the VSE. These are listed in Table 3. HSS responds to 16 risks defined in the BCPR. Each risk is further decomposed by Enabling Questions (EQ). The enabling questions pose the research areas needed to mitigate each risk. The HSS Program addresses a total of $150 \mathrm{EQs}$.

\section{Gap Analysis}

Management of the HSS Program to respond to a dynamic programmatic environment requires periodic assessment to maintain an appropriate balance and set of priorities across all of the research and technology development tasks. Program management must evaluate the entire set of tasks to ensure that gaps and overlaps in the technical work are identified and are reduced to an acceptable degree. The HSS Program portfolio tool is a graphical method to illustrate the program technical content, allowing managers to easily view ongoing work by area, assist project selection, assess gaps, and discern areas of overlap between tasks. Fig. 1 provides an example of the tool interface.

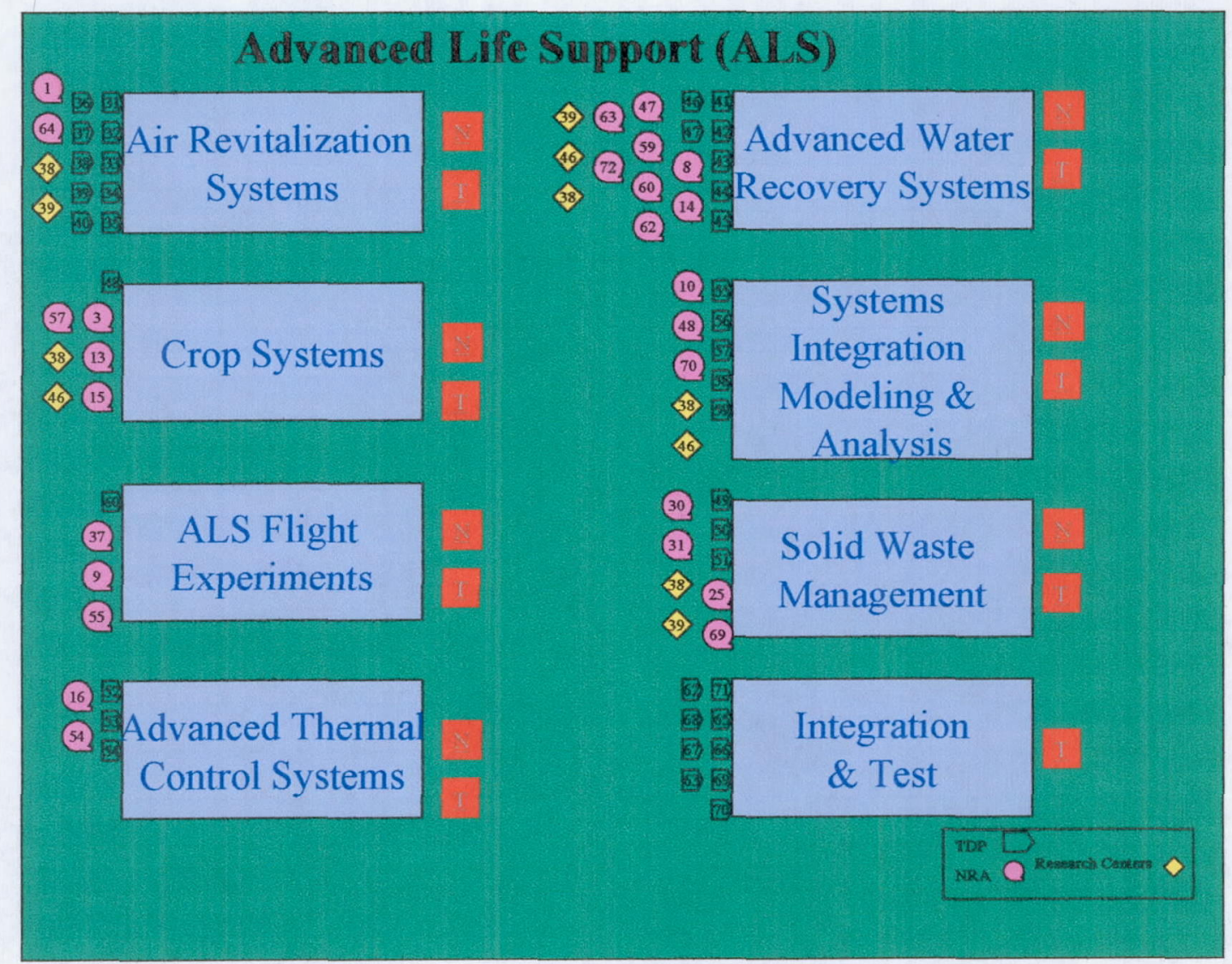

Figure 1. HSS Program Portfolio Mapping Tool 


\section{Program Portfolio Strategy}

The program portfolio is driven by the nine-point Technology Readiness Level (TRL) scale used by NASA which is shown in Table 4. ${ }^{9}$ Table 4. NASA Technology Readiness Levels.

HSS technical tasks, in general, TRL 1 Basic principles observed and reported raise technologies to TRL 6 , thereby mitigating much of the risk associated with them and encouraging exploration programs to accept them into their design trade studies and systems engineering efforts. The research and development is accomplished by broad collaboration with experts in academia, other agencies, industry, and within NASA TRL 2 Technology concept and/or application formulated

TRL 3 Analytical and experimental critical function and/or characteristic proof-of-concept

TRL 4 Component and/or breadboard validation in laboratory environment TRL 5 Component and/or breadboard validation in relevant environment

TRL 6 System/subsystem model or prototype demonstration in a relevant environment (ground or space)

TRL 7 System prototype demonstration in a space environment

TRL 8 Actual system completed and "flight qualified" through test and demonstration (ground or space)

TRL 9 Actual system "flight proven" through successful mission operations through a combination of:

(1) Search for innovation by investigation of applicable emerging, potentially substantially better technology solutions to the EQs. This is accomplished by frequent interactions with the external research community through conferences and open solicitations. A portfolio of peer reviewed, hypothesis driven proposals and the small business innovative research processes are selected based on scientific merit, program relevance, and feasibility of implementation. These are externally managed by the investigator, with regular, frequent interactions with element management to assure investigations.

(2) Evolutionary advances in existing technologies, integration and technology maturation. These are internally managed development efforts, using both intramural and extramural resources.

(3) Development of operational concepts that utilize the best features of these systems through simulation, installation of prototypes in analog environment, and working with the experienced human operations community.

(3) Methodical testing and interface management through all stages of system development

\section{E. Program Portfolio Solicitation, and Selection}

The NASA Broad Area Announcement (BAA) mechanism is used as one solicitation method for announcements to both of these communities. It provides guidance on the required technical focus for task proposals to HSS. Past NASA Research Announcements (NRAs) have often allowed proposals to develop technologies at low TRL, in the laboratory setting. A recent NRA used the Rapid Technology Development Team (RTDT) approach to solicit proposals for mid-TRL work that can bridge the gap between laboratory research and NASA applications. RTDTs bring together extramural and intramural team members to accomplish rapid development and delivery of technologies that can have an immediate impact in meeting the HSS Program's needs.

The technology maturation process is directed work targeted to meet specific requirements to raise technologies to mid- or higher-TRLs for integration, testing, or flight demonstration. This work is accomplished using teams of external researchers, NASA staff, and competitively selected support contractors.

\section{F. Progress Measurement}

The HSS Program measures its progress in several ways. The BCPR is used as the basis for metrics associated with the results of individual technical tasks. Annual reviews at both the task level and the program element level are used to determine how effectively various aspects of the program are being managed.

Fig. 2 illustrates the technical task feedback that enables closed-loop guidance of the HSS portfolio. 


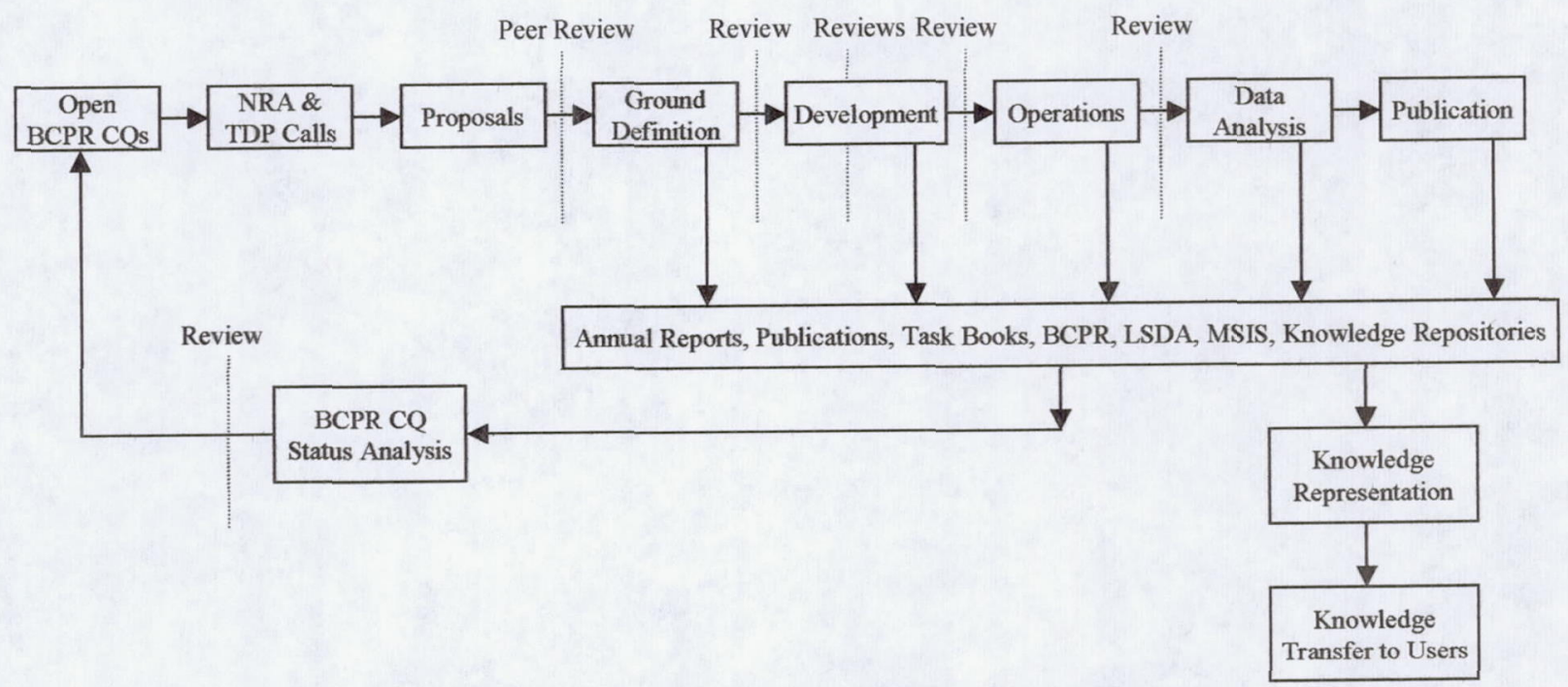

Figure 2. BCPR-Driven Technical Tasks and Feedback

\section{G. Product and Knowledge Management}

HSS technical tasks result in deliverable products of new knowledge, prototype software and hardware, and systems studies. The BCPR Enabling Questions were used to generate the HSS Product Breakdown Structure (PBS). This PBS covers all identified deliverables that are required to respond to the EQs. These items are captured during the various phases of the HSS technical tasks, as shown in Fig. 2 above. Compilation, integration, and review of these products allow the closed-loop process to continually align HSS efforts. It also spawns major HSS Program outputs in the form of knowledge representation and transfer to users.

Much of the scientific and technological knowledge gained from HSS Program activity is captured in NASA design standards, for the benefit of user programs that create mission and system designs. Current examples include NASA Standard 3000, the Man-Systems Integration Standards. ${ }^{10}$

The HSS Program's major outputs are transferred to the NASA Space Architect Team, the Human \& Robotic Technology Program, the ExSD Requirements Division, and the ISS Program. HSS also coordinates its efforts with other NASA areas, including the Aeronautics Program and the NASA Chief Engineer, who manages all technical standards.

\section{Systems Engineering and Integration}

Experience in space flight programs has demonstrated the value of early systems engineering and integration and ground based testing before deployment of a space-based system with very limited capability for resupply or replacement. The HSS Program has implemented a multi-element systems engineering and integration function, to provide a foundation for demonstration of its technologies. This process involves formulation of systems studies, integrated testing, validation of integration requirements, and interaction across the HSS elements. The Advanced Integration Matrix (AIM) team leads the systems engineering effort for the HSS program. ${ }^{11}$

\section{A. Systems Engineering}

The distributed nature of the HSS development necessitates a strong capability to determine how a component performs within a subsystem, and how the subsystem performs in the complex interactions of systems and the humans operating them. The complexity of the HSS development effort drives the need for a systems approach. A systems integration process addressing HSS issues yields validation of advanced concepts and technologies, establishes baselines for further development, and identifies opportunities for system-level advancements. Where appropriate, lessons learned from previous programs including Skylab, the International Space Station, and the Space Shuttle are considered and applied to HSS efforts. The tools that are used to support the integration of human support systems include trade studies and analyses, integrated systems models, and integrated system testing. Models are used to evaluate interface designs, to predict integration failures, and to baseline the performance of human support systems, for example, using probabilistic risk assessment techniques. Trade study topics are identified and prioritized for implementation. The results from these studies influence and validate design concepts, 
generate topics for future trade studies, and identify candidates for integrated testing. Fig. 3 illustrates the AIM trade study process. Examples of potential trade studies include mechanisms for dust abatement during planetary surface extravehicular activity, crop growth options, food processing techniques, and habitat radiation shielding by use of life support systems and crew consumables.

\section{B. Systems Integration and Test}

NASA has used ground-based technology integration and testing in previous programs to contribute to their success. The Skylab Medical Experiments Altitude Test, Space Shuttle Integrated Environmental Control and Life Support Systems (ECLSS) testing, and ISS ECLSS testing provided a degree of pre-mission risk mitigation for each of these

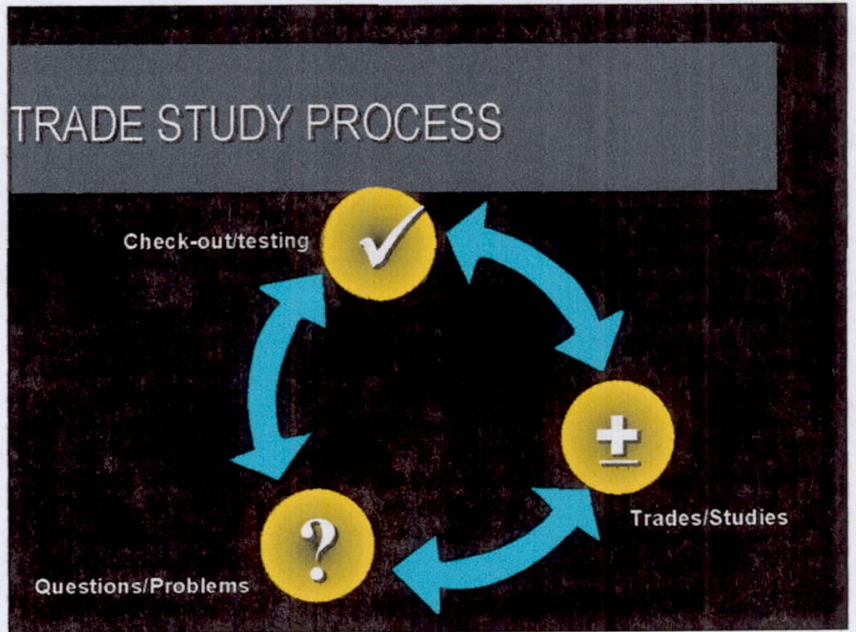

Figure 3. AIM Trade Study Process. programs. In addition, integrated testing, often with human subjects, has been used by NASA to raise the readiness levels of certain technologies. The Lunar Mars Life Support Test Project integrated tests were performed for this purpose.

Ground based integrated research and testing identifies requirements and reveals undesired emergent system behavior prior to flight, prompting refinement and validation of requirements and solutions, and supports a safe, cost-effective risk mitigation strategy for human exploration. Early ground-based testing of mission capability identifies successful system implementations and operations, hidden risks and hazards, unexpected system and operations interactions, mission mass and operational savings, and can evaluate solutions to requirements-driving questions; all of which enable HSS and its customer programs to develop more effective, lower risk systems and more reliable cost estimates for planetary exploration missions. It also drives early validation of human-rating requirements, human engineering, and crew systems, and validates interfaces to identify areas of potential commonality across HSS systems.

Examples of system level integration and interface issues that are evaluated during ground based testing include: sparing; system mass reduction; commonality of hardware, interfaces, and procedures; automation levels; maintenance and repair; reliability and redundancy requirements; crew size and skill mix; failure recovery modes; and vehicle environmental monitoring and control.

The process for determination of which HSS components will be tested when and where is under development. Ground testing and analysis will be employed to promote the TRL of HSS systems, by providing the means to demonstrate technologies in relevant test environments. HSS ground based testing will engage resources available at many of the NASA centers, using an appropriate set of facilities. In each instance the test location will be chosen based on the specific hardware or software to be tested, test requirements, personnel requirements, and facility availability. The methodology for producing the program integration schedule will utilize matrices of project characteristics based on comparable readiness of the technologies to be integrated, the level of interaction of the various technologies, and driven by the need dates of the ExSD flight program.

\section{In-Space Research and Testing}

HSS uses the ISS as a microgravity testbed for human research, physical research, and testing of technological prototypes. The Bioastronautics Flight Research Program acts as the flight integration function for HSS flight experiments developed at multiple NASA and external sites.

In-space human research is performed in areas such as EVA technologies, food technologies, and space human factors engineering, to discover the effects of microgravity on specific aspects of human performance and to gather data necessary for the future design of planetary exploration missions and systems. ISS research infrastructure such as the Human Research Facility is used to enable this research.

Physical research into the characteristics of spacecraft materials, including the flammability of non-metallic substances and the behavior of multi-phase fluid systems, is performed on ISS to provide a basis for future spacecraft and life support system designs. Research infrastructure onboard ISS such as the Fluids and Combustion Facility is used to enable this effort. 
Technology testing, including new methods of life support and environmental monitoring, is performed on ISS, supported by facilities such as the Expedite the Processing of Experiments for Space Station (EXPRESS) rackmounting accommodations for modular payloads.

HSS payloads are currently in all life-cycle stages: early conceptual definition, hardware and software development, testing and integration for launch, and operation onboard the ISS. These include the flight experiments listed in Table 5. HSS cooperates with the Russian space flight community on experiments that are hosted in the ISS Russian Segment. HSS is also cooperating with the European Space Agency to launch and operate the Analyzing Interferometer for Ambient Air payload on ISS.

\section{Technology Integration}

To complement the technical expertise within the elements of the program, HSS utilizes a Technology Integration Agent (TIA) function to take advantage of non-NASA research efforts. The TIA provides a broad view of the technological horizon outside NASA, to bring external knowledge and technologies into the HSS Program when advantageous. TIA tasks are identified through interactions with the HSS elements, based on their needs for specific solutions to a technology need, and based on the TIA's assessment of non-NASA work applicable to the domain under consideration. The TIA retains subject matter experts from industry, academia, or partnering organizations, such as military funded programs, as required to support each specific project.

This cost effective project management model allows the HSS program to make a small investment in technology watch activities that provides it a valuable degree of insight into a wide spectrum of technological areas. It creates opportunities to leverage external technology development activities to the benefit of HSS and its customer programs.

The practical Table 5. Current ISS Flight Experiments

implementation of the Optimization of Root Zone Substrates for Reduce Gravity Experiments

TIA process was Root Modules

demonstrated when the

TIA provided the HSS Water Offset Nutrient Delivery Experiment

Program a set of Sabatier Carbon Dioxide Reduction Assembly

immediately available $\quad$ Microgravity Investigation of Crew Reactions

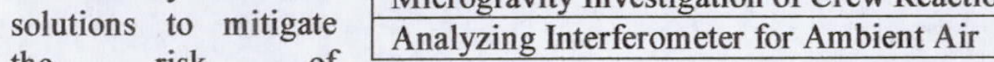

the risk of

environmental hazards onboard the ISS. A database of commercially available environmental monitoring hardware was delivered by the TIA to the HSS program. As a follow-on effort, the ISS Program released a formal Request for Information (RFI) for the purpose of identifying second-generation environmental monitoring systems with a demonstrated ability or potential to meet defined requirements for systems to ensure air and water quality on-board the ISS. The TIA implemented this RFI effort to the benefit of HSS and the ISS Program.

\section{Conclusion}

Technologies developed by the HSS Program are essential to NASA's future exploration missions. HSS has aligned itself to the Vision for Space Exploration, with the focus of providing critical technologies for human exploration of space. To ensure program success, HSS utilizes best practices in project management and systems engineering.

\section{Acknowledgments}

The authors are grateful for the support of the ExSD, the HSS Program element and field center offices, and their staff members, whose participation in the processes described here are vital to the effective management of the program.

\section{References}

${ }^{1}$ Committee on Advanced Technology for Human Support in Space, Aeronautics and Space Engineering Board, Commission on Engineering and Technical Systems, National Research Council, "Advanced Technology for Human Support in Space," National Academies Press, 1997.

${ }^{2}$ National Aeronautics and Space Administration, "NASA Strategic Plan," 2003.

${ }^{3}$ National Aeronautics and Space Administration, "Biological and Physical Research Enterprise Strategy," 2003.

4National Aeronautics and Space Administration, "Bioastronautics Strategy," Final Version, January 27, 2003.

${ }^{5}$ National Aeronautics and Space Administration, "The Vision for Space Exploration," February 2004. 
${ }^{6}$ Aldridge, E. C., et al, "A Journey to Inspire, Innovate, and Discover", Report of the President's Commission on Implementation of United States Space Exploration Policy, June 2004.

${ }^{7}$ National Aeronautics and Space Administration, "Draft Bioastronautics Critical Path Roadmap (BCPR), An Approach to Risk Reduction and Management for Human Space Flight: Extending the Boundaries," JSC-62577, April 2, 2004.

${ }^{8}$ National Academies Institute of Medicine, "Review of NASA's Bioastronautics Critical Path Roadmap," URL: http://www4.nas.edu/webcr.nsf/ProjectScopeDisplay/HSPX-H-04-01-A [cited June 21, 2004].

'Mankins, J. C., "Technology Readiness Levels: A White Paper", NASA, April 6, 1995.

${ }^{10}$ National Aeronautics and Space Administration, "Man-Systems Integration Standards", NASA STD-3000 Revision B, July 1995.

${ }^{11}$ Paul, Heather, "Advanced Integration Matrix Education Outreach," International Conference on Environmental Systems 2004-01-2481, SAE International, July 2004. 